\title{
One-way multi-party communication lower bound for pointer jumping with applications
}

Emanuele Viola*

\author{
Avi Wigderson ${ }^{\dagger}$
}

July 23,2007

\begin{abstract}
In this paper we study the one-way multi-party communication model, in which every party speaks exactly once in its turn. For every fixed $k$, we prove a tight lower bound of $\Omega\left(n^{1 /(k-1)}\right)$ on the probabilistic communication complexity of pointer jumping in a $k$-layered tree, where the pointers of the $i$-th layer reside on the forehead of the $i$-th party to speak. The lower bound remains nontrivial even for $k=(\log n)^{1 / 2-\Omega(1)}$ parties. Previous to our work a lower bound was known only for $k=3$ [BHK], and in very restricted models for $k>3$ [DJS, Cha]. Our results have the following consequences to other models and problems, extending previous work in several directions.

The one-way model is strong enough to capture general (non one-way) multi-party protocols of bounded rounds. Thus we generalize to this multi-party model results on two directions studied in the classical 2-party model (e.g. [PS, NW]). The first is a round hierarchy: We give an exponential separation between the power of $r$ and $2 r$ rounds in general probabilistic $k$-party protocols, for any fixed $k$ and $r$. The second is the relative power of determinism and nondeterminism: We prove an exponential separation between nondeterministic and deterministic communication complexity for general $k$-party protocols with $r$ rounds, for any fixed $k, r$.

The pointer jumping function is weak enough to be a special case of the well-studied disjointness function. Thus we obtain a lower bound of $\Omega\left(n^{1 /(k-1)}\right)$ on the probabilistic complexity of $k$-set disjointness in the one-way model, which was known only for $k=3$ parties. Our result also extends a similar lower bound for the weaker simultaneous model, in which parties simultaneously send one message to a referee [BPSW].

Finally, we infer an exponential separation between the power of different orders in which parties send messages in the one-way model, for every fixed $k$. Previous to our work such a separation was only known for $k=3[\mathrm{NW}]$.

Our lower bound technique, which handles functions of high discrepancy, may be of independent interest. It provides a "party-elimination" induction, based on a restricted form of a direct-product result, specific to the pointer jumping function.
\end{abstract}

*The author is supported by NSF grant CCR-0324906.

${ }^{\dagger}$ The author is supported by NSF grant CCR-0324906. 